\title{
Temperature effects on optical properties and efficiency of red AIGalnP-based light emitting diodes under high current pulse pumping
}

Amit Yadav, Ilya E. Titkov, Grigorii S. Sokolovskii, Sergey Yu. Karpov, Vladislav V. Dudelev, Ksenya K. Soboleva, Martin Strassburg, Ines Pietzonka, Hans-Juergen Lugauer, and Edik U. Rafailov

Citation: Journal of Applied Physics 124, 013103 (2018); doi: 10.1063/1.5020266

View online: https://doi.org/10.1063/1.5020266

View Table of Contents: http://aip.scitation.org/toc/jap/124/1

Published by the American Institute of Physics

\section{Articles you may be interested in}

Diode junction temperature in ultraviolet AIGaN quantum-disks-in-nanowires

Journal of Applied Physics 124, 015702 (2018); 10.1063/1.5026650

Alloy disorder limited mobility of InGaN two-dimensional electron gas

Applied Physics Letters 112, 262101 (2018); 10.1063/1.5030992

Experimental characterization of impact ionization coefficients for electrons and holes in GaN grown on bulk GaN substrates

Applied Physics Letters 112, 262103 (2018); 10.1063/1.5031785

Band alignment of $\mathrm{AIN} / \mathrm{\beta}-\mathrm{Ga}_{2} \mathrm{O}_{3}$ heterojunction interface measured by $\mathrm{x}$-ray photoelectron spectroscopy

Applied Physics Letters 112, 261602 (2018); 10.1063/1.5035372

Prediction of multiband luminescence due to the gallium vacancy-oxygen defect complex in GaN

Applied Physics Letters 112, 262104 (2018); 10.1063/1.5026751

Structural and electronic properties of a-edge dislocations along $\langle 1-100\rangle$ in GaN

Journal of Applied Physics 123, 244301 (2018); 10.1063/1.5034198

\section{AIPP| Iopplied f Physics SPECIAL TOPICS}

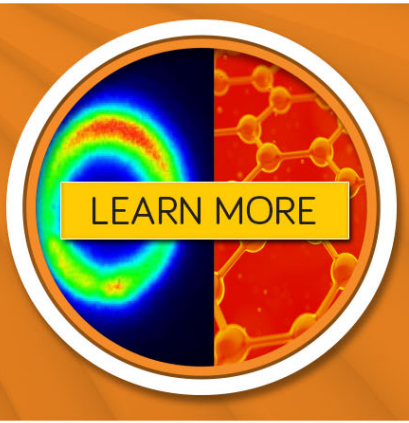




\title{
Temperature effects on optical properties and efficiency of red AIGalnP-based light emitting diodes under high current pulse pumping
}

\author{
Amit Yadav, ${ }^{1, a)}$ llya E. Titkov, ${ }^{2}$ Grigorii S. Sokolovskii, ${ }^{3,4}$ Sergey Yu. Karpov, ${ }^{5}$ \\ Vladislav V. Dudelev, ${ }^{3}$ Ksenya K. Soboleva, ${ }^{4}$ Martin Strassburg, ${ }^{6}$ Ines Pietzonka, ${ }^{6}$ \\ Hans-Juergen Lugauer, ${ }^{6}$ and Edik U. Rafailov ${ }^{1,7}$ \\ ${ }^{1}$ Aston Institute of Photonic Technologies, Aston University, B4 7ET Birmingham, United Kingdom \\ ${ }^{2}$ Ostendo Technologies, Inc., 6185 Paseo del Norte, Carlsbad, California 92011, USA \\ ${ }^{3}$ Ioffe Institute, 26 Polytekhnicheskaya str., 194021 St. Petersburg, Russia \\ ${ }^{4}$ St. Petersburg State Electrotechnical University (LETI), 5 Prof. Popova str., 197022 St. Petersburg, Russia \\ ${ }^{5}$ STR Group - Soft-Impact, Ltd., P.O.Box 83, 27 Engels ave., 194156 St. Petersburg, Russia \\ ${ }^{6}$ OSRAM Opto Semiconductors GmbH, Leibnitzstr. 4, 93055 Regensburg, Germany \\ ${ }^{7}$ Saratov State University, International Center of Critical Technologies in Medicine, Astakhanskaya Str. 83, \\ Saratov, 410012, Russia
}

(Received 21 December 2017; accepted 18 June 2018; published online 6 July 2018)

\begin{abstract}
In this paper, current-dependent emission spectra and efficiency measured on the same AlGaInP red light-emitting diode (LED) pumped with the current pulses of very different durations are recorded. This enabled for the first time distinguishing between high-carrier concentration and selfheating effects on the efficiency decline at high current magnitudes. The electron leakage to the pside of the LED structure, which is the major mechanism of the efficiency reduction, is found to rise substantially when the device self-heating starts to develop. As a result, in comparison to continuous-wave excitation, driving the LED with sub-microsecond current pulses allows suppressing the device self-heating and, eventually, increasing the operating current by an order of magnitude without noticeable efficiency losses. Based on the reduced ABC-model, neglecting Auger recombination, the light extraction efficiency, injection efficiency, and internal quantum efficiency of the LED are estimated, suggesting light extraction to be the most critical factor for the overall efficiency of the LED. The coupled spectral/power LED characterization using the variableduration current pulse pumping is found to be an effective approach for analyzing mechanisms of the device operation. Published by AIP Publishing. https://doi.org/10.1063/1.5020266
\end{abstract}

\section{INTRODUCTION}

Development of light-emitting diodes (LEDs) has gained significant impulse and attention due to their promise of better light quality and emission efficiency. They are being used in numerous applications such as signage, construction signs, surgical lighting, diagnostic equipment, automotive applications, and solid-state lighting (SSL). ${ }^{1,2}$ A multichip approach combining red, green, and blue LEDs is one of the promising methods to generate white light for SSL, especially with a significant increase in luminous efficacy of both III-Nitride and III-Phosphide LEDs over the last decade. ${ }^{3}$

III-Phosphide based (AlGaInP) LEDs emit in the wavelength region of $560-650 \mathrm{~nm}$ of the visible spectrum where the emission wavelength is determined by the aluminum (Al) content in the quaternary alloy. ${ }^{1,4}$ While the peak emission can be tuned towards the green region of the spectrum with increasing $\mathrm{Al}$ content, it also leads to reduced LED emission efficiency. This dramatic efficiency decrease with the wavelength has been attributed to carrier escape from the quantum-well $(\mathrm{QW})$ active region to indirect-bandgap confining barriers. ${ }^{5,6}$ The same effect is believed to be responsible for substantial reduction of external quantum efficiency

${ }^{a)}$ Electronic mail: a.yadav1@aston.ac.uk.
(EQE) of AlGaInP based red LEDs with driving current, ${ }^{7}$ which is a limitation to reduce cost per lumen as well as for high brightness applications. On the other hand, an alternative explanation for the efficiency reduction can be found in the literature. Based on the electrical-to-optical pulse response method, Shi et al., suggested defect recombination and saturated spontaneous recombination at low $(<16.7 \mathrm{~A} /$ $\left.\mathrm{cm}^{2}\right)$ and high $\left(>27.8 \mathrm{~A} / \mathrm{cm}^{2}\right.$ to $\left.100 \mathrm{~A} / \mathrm{cm}^{2}\right)$ current densities, respectively, to be responsible for the efficiency decrease in red LEDs. ${ }^{8}$ The electron leakage induced by asymmetry in the p-n junction at low currents has been considered as a contributor towards the efficiency decrease in Refs. 9 and 10. In contrast, Windisch et al. attributed the carrier leakage to lower barrier heights in the confinement layers between $\mathrm{QWs},{ }^{11}$ which is the mechanism very close by its nature to the escape-based one proposed in Refs. 5-7. The importance of the mechanism has been convincingly argued by evaluation of the carrier leakage activation energy being almost equal to the QW confinement energy, i.e., the energy difference between the QW and the confinement layers and controlling a strong temperature dependence of the leakage. ${ }^{12} \mathrm{In}$ light of these arguments, similar effects on the efficiency reduction must be observed with rising of the electron quasiFermi level due to increased carrier concentration in QWs and the increase in the high energy electron density due to LED self-heating. It is also well understood that both, the 
radiative recombination constant and non-radiative carrier lifetime, decrease with temperature. So, the LED efficiency may decrease by itself without any contribution from the carrier leakage when operated at high temperatures. It is still an open question despite numerous investigations of red LEDs. Which of the effects, (a) the effect of elevated temperature or (b) of high carrier concentration in the active region, produces the major contribution to the LED efficiency declining at high currents. The impacts of the above factors are hardly distinguishable under continuous-wave $(\mathrm{CW})$ operating conditions but they can be resolved at pulsed-current excitation where the pulse duration controls the cooling rate of the LED active region.

Indeed, LED excitation by a current pulse with a constant magnitude provides self-heating of the device active region with the rate of heat release proportional to the magnitude. On the other hand, the rate of the heat removal (heat flux) from the active region decreases with time, being proportional to the monotonously decaying temperature gradient. Therefore, at a given magnitude of current, the temperature of the active region decreases with the current pulse duration, so that shorter pulses produce a lower LED self-heating. Intentional variation of the pulse duration, keeping the current magnitude unchanged, enables one either to enhance or to suppress the thermal effects, while the carrier concentration in the active region is still controlled by the magnitude of current.

From the practical point of view, improvement of LED efficiency at high current densities is quite desirable for many applications. In SSL, this would increase the radiant flux obtained from the unit area of the wafer, which is important for cost reduction. ${ }^{2,3}$ In some other particular applications like micro-LEDs, this is just a vital issue, as such devices operate principally at the current densities much exceeding those of conventional LEDs. ${ }^{13,14}$ Due to suppression of current crowding, the micro-LEDs exhibit unexpectedly efficient heat removal from the active region. ${ }^{13,15}$ Therefore, distinguishing between the thermal and high carrier-concentration effects would be a proper basis for optimization of their efficiency.

It should be noted that the use of electric-pulse excitation of LEDs aimed at suppression of thermal effects may be a specific way for improvement of their efficiency. In particular, its application to III-nitride micro-LEDs has provided dramatic, by an order of magnitude, improvement of their high-current efficiency when the devices were pumped by nanosecond current pulses. ${ }^{16}$ To our knowledge, there are no similar studies carried out with red LEDs.

One of the goals of the study reported in this paper is distinguishing between the thermal and high-carrier concentration effects on the efficiency reduction of AlGaInP LEDs at high current densities. For this purpose, emission efficiency and spectra as a function of current magnitude obtained from the same LED pumped by current pulses of very different durations are compared for the first time. A commercial red LED emitting at $620 \mathrm{~nm}$ has been examined under different excitation conditions, namely, in continuous wave $(\mathrm{CW})$ and pulse regimes, ranging from microsecond to sub-nanosecond current pulses, and at very high currents up to $45 \mathrm{~A}$ at $10 \mathrm{kHz}$ repetition rate with sub-nanosecond pulses. The use of the variable current pulse duration enabled the control of the heat removal from the active region, thus influencing the interplay between high-carrier concentration and thermal effects. ${ }^{17}$ Another goal, which is important for practice, is the assessment of possible improvements of high-current LED efficiency by using pulsed-current excitation of various durations. In this way, we had to estimate separately light extraction efficiency (LEE), injection efficiency (IE), and internal quantum efficiency (IQE) of the studied LED that altogether determined the overall efficiency of the device.

Methodologically, we have chosen optical characterization of LEDs in our study, allowing simultaneous monitoring of both spectral and power device characteristics. Our study focuses on finding correlations between the LED emission spectra and EQE behavior as a function of current, which enables distinguishing between the high-carrier concentration and self-heating effects. The data on the LED efficiency are interpreted within the AB-recombination model considering interplay of only two principal, non-radiative, and radiative, recombination channels ${ }^{18}$ (see the Appendix). In the absence of carrier leakage from the LED active region, the model predicts the analytical dependence of its $E Q E \eta_{e}$ on the output optical power $P_{\text {out }}{ }^{19}$

$$
\eta_{e}\left(P_{\text {out }}\right)=\eta_{\text {ext }} P_{\text {out }}^{1 / 2} /\left(P_{\text {out }}^{1 / 2}+P_{0.5}^{1 / 2}\right),
$$

where two parameters, the light extraction efficiency $\eta_{\text {ext }}$ and the power $P_{0.5}$, can be found from fitting the measured dependence $\eta_{e}\left(P_{\text {out }}\right)$ at sufficiently low operating currents (see Ref. 19 for more details). Evaluation of LEE $\eta_{\text {ext }}$ allows making breakdown of the efficiency losses and identifying bottlenecks for achieving the ultimate LED performance.

\section{DEVICE STRUCTURE}

The AlGaInP high power LEDs investigated in this study are grown on (001) GaAs substrates by metal organic vapor phase epitaxy (MOVPE) in an industry scale multiwafer reactor. The LED structure consists of 20 undoped multiple $\left(A l_{x} G a_{(1-x)}\right)_{0.52} \operatorname{In}_{0.48} P$ quantum wells (MQWs) in the active layer with a total thickness of $280 \mathrm{~nm}$. The MQWs are embedded between $\mathrm{n}$ - and p-doped $A l_{0.52} I_{0.48} P$ confinement layers. The aluminum content, $\mathrm{x}$, of the active region is adjusted to approximately $12 \%-16 \%$, to achieve the emission spectrum to peak at nearly $620 \mathrm{~nm}$. The LED heterostructure was completed with the p-AlGaAs spreading layer and $\mathrm{p}^{+}-\mathrm{GaAs}$ contact layer. The grown structure was etched through the $\mathrm{p}-\mathrm{AlGaAs} / \mathrm{p}^{+}-\mathrm{GaAs}$ spreader/contact layer to form ordered frustums of pyramids serving as microreflectors embedded into the LED die. After subsequent deposition of the p-electrode and the dielectric/metal mirror, the wafer was soldered onto a GaAs carrier wafer by metal-bonding. Then, the GaAs substrate was removed by selective etching, leaving the epitaxial LED structure to sit on the new carriersubstrate with a highly reflective mirror in between. The die processing by the OSRAM OS's standard Thinfilm ${ }^{\mathrm{TM}}$ technology was finalized by formation of n-electrodes and surface texturing between them to enhance the light extraction. The ThinFilm technology and schematic chip design are described 
in detail in Ref. 11. Finally, single $1 \times 1 \mathrm{~mm}^{2}$ LED chips were fabricated from the wafer before packaging into the Golden Dragon cases.

\section{EXPERIMENTAL DETAILS}

The LEDs are studied under both the continuous wave (CW) and pulsed regime with an integration sphere. To determine the absolute values of $\mathrm{EQE}$ at room temperature, the light output from the integration sphere is collected with a Labsphere "CDS-600 spectrometer" and the data are processed by the "LightMtrx" software to determine radiant flux, peak wavelength, and full bandwidth at half maximum (FWHM). To operate the LED under the $\mathrm{CW}$ regime, a "Keithley 2400" source with a maximum output current of $1 \mathrm{~A}$ is used. Next, the "IXYS Colorado PCX-7420" pulse laser diode driver with external triggering is used to drive the LED in the current range of 2-23 A with sub-microsecond pulses.

Furthermore, a custom made pulse generator designed to generate sub-nanosecond, i.e., $0.7 \mathrm{~ns}$ pulses at very high currents up to $45 \mathrm{~A}$ is employed. In a wide range of pulse widths used in experiments, the electroluminescence (EL) is recorded with photo detectors and oscilloscopes with different rise times and bandwidths (BW) for sub-microsecond and sub-nanosecond pulses. While an oscilloscope with $1 \mathrm{GHz}$ BW is used for sub-microsecond EL measurements, a $50 \mathrm{GHz}$ BW "Agilent 86100A" oscilloscope is used for the sub-nanosecond regime. Similarly, a photo detector with a broad $\mathrm{BW}$ of $30 \mathrm{GHz}$ and a photo detector with $14 \mathrm{~ns}$ rise time are used in sub-nanosecond and sub-microsecond regimes, respectively. To avoid the thermal effect of a current pulse on the next one, the duty cycle is chosen carefully to be $\leq 1 \%$.

It is crucial for the methodology presented here to maintain the pulse amplitude due to non-linear impedance and thus, a non-contact TCP0030A AC/DC current probe is used to monitor it on the oscilloscope. Finally, to account for the effect of temperature on peak wavelength a "Janis CCS450"closed cycle Helium cryostat is used to achieve low temperatures for temperature dependent electroluminescence (TDEL) measurements.

\section{RESULTS AND DISCUSSION}

\section{A. Pulse width and spectral evolution}

The room-temperature CW current-voltage characteristics of the studied LEDs could be well fitted by the Shockley's diode equation with the ideality factor of 1.3 and series resistance of $1.15 \mathrm{Ohm}$. Exactly the same parameters were extracted from the current-voltage characteristics measured with $100 \mu$ s current pulses. Their values certify optimized electrical design of both the LED structures and chip. The inset in Fig. 1(a) shows the micrograph of the LED chip operating at $1 \mathrm{~A} \mathrm{CW}$. The picture demonstrates a rather uniform emission pattern over the whole chip area except for a
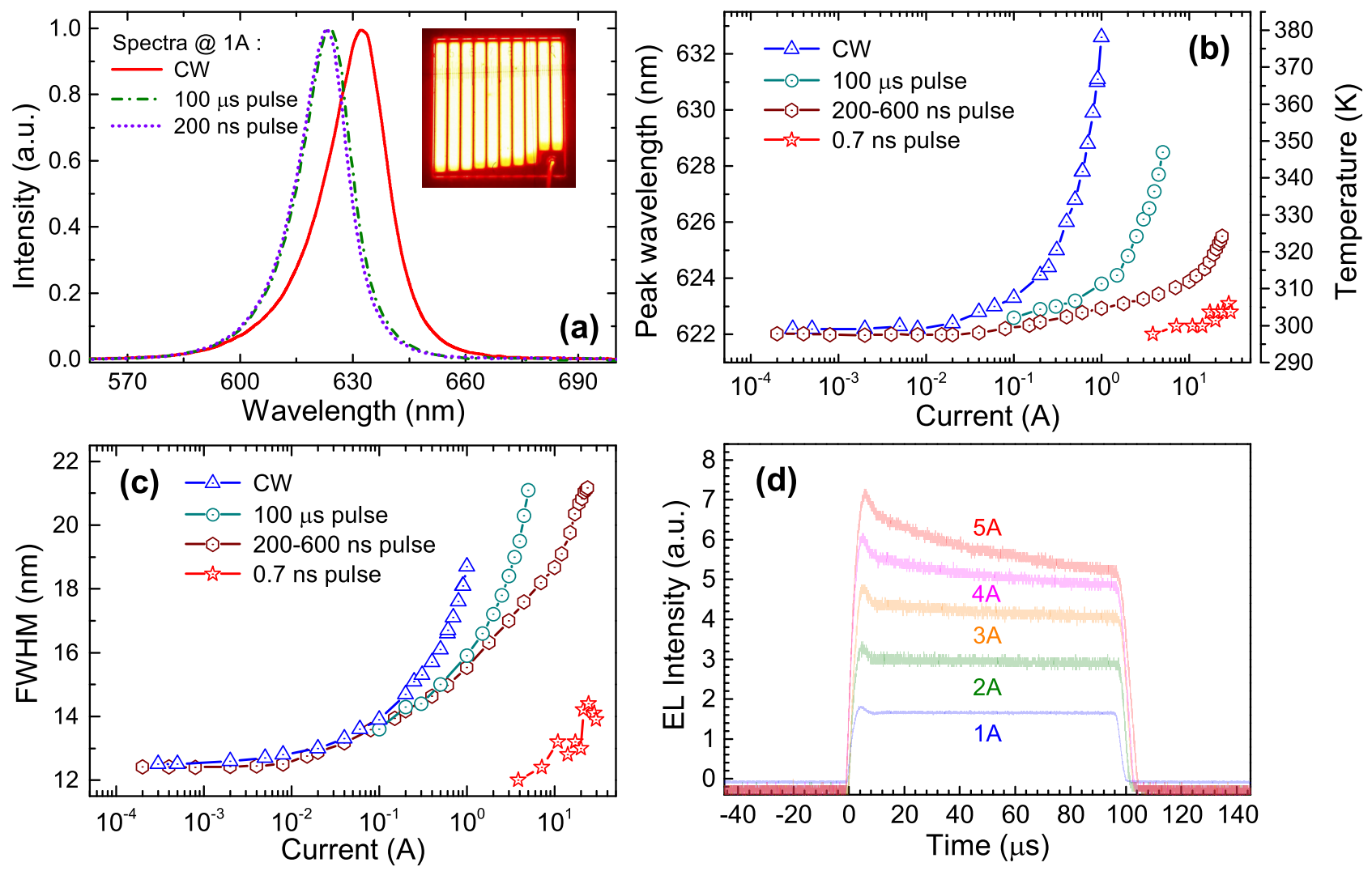

FIG. 1. (a) EL emission spectra under CW, $100 \mu$ s and 200-600 ns pulses at 1\% duty cycle for 1 A current, (b) peak wavelength vs current at various operation conditions and corresponding temperature (right vertical) scale, (c) FWHM of the emission spectrum as a function of current at CW (triangles), $100 \mu$ s (circles), 200-600 ns (hexagon), and $0.7 \mathrm{~ns}$ (stars) pulse excitation, and (d) time-resolved EL intensity under $100 \mu$ s pulse excitation at different magnitudes of current. The inset in (a) shows a micrograph of the LED operating at $1 \mathrm{~A} \mathrm{CW}$ (see the text for more details). The temperature scale in plot(b) is calibrated by $\mathrm{CW}$ measurements of the red spectral shift at various temperatures (see last paragraph of Sec. IV A for more details). 
narrow region adjacent to the bottom contact electrode where current crowding produces some non-uniformity. Since the area of this region is remarkably smaller than the whole area of the chip, we believe that current crowding does not interfere with both the emission spectra and the LED efficiency at high currents.

The emission spectrum of the red LED at $1 \mathrm{~A}$ of current under the CW and pulsed regime, for both $100 \mu$ s and $200 \mathrm{~ns}$ pulses, is shown in Fig. 1(a) depicting a clear shift in emission wavelength under different pumping conditions. The EL spectra of the LED when excited with $100 \mu$ s pulses at $1 \%$ duty cycle in the current range of $100 \mathrm{~mA}$ to $5 \mathrm{~A}$ show a shift of $\sim 6 \mathrm{~nm}$ in the emission peak towards the longer wavelengths, i.e., a red shift, ${ }^{17}$ as is evident from Fig. 1(b). A similar, $\sim 6-7 \mathrm{~nm}$, red shift is observed when the device is operated in the current range of $10 \mathrm{~mA}-600 \mathrm{~mA}$ in the $\mathrm{CW}$ regime [see Fig. 1(b)].

It is also noted that the red shift is rather small (less than $\sim 2 \mathrm{~nm}$ ) at $100 \mu$ s pulses for current magnitudes up to $\sim 1 \mathrm{~A}$. However, further increase in current to $5 \mathrm{~A}$ leads to a substantial red shift of $6 \mathrm{~nm}$ [see Fig. 1(b)] and spectral broadening of $\sim 8.5 \mathrm{~nm}$ [Fig. 1(c)], which is two times larger than the broadening of $\sim 4 \mathrm{~nm}$ observed at $1 \mathrm{~A}$. This observation correlates with the increasing slope of the high energy part of the emission spectrum at high currents, which is similar to that reported in Ref. 10 and can be attributed to a rise in temperature of the non-equilibrium carriers. Next, the EL intensity during the $100 \mu$ s pulse is examined as a photodiode response in the current range from $100 \mathrm{~mA}$ to $5 \mathrm{~A}$. A substantial decrease in the EL intensity during the pulse action can be observed at currents $\sim 2$ A [Fig. 1(d)] getting more pronounced with increasing magnitude of current. This is the evidence for the gradual (comparable with the current pulse duration) increase in temperature in the active region, resulting in the efficiency decline. Such a transient behavior was not visible at the currents lower than $1 \mathrm{~A}$.

Furthermore, Fig. 1(b) also shows that the observed red shift has a distinctive two-slope character: slow growth at low currents and fast increase at high currents. The onset of the red shift at $\sim 10-30 \mathrm{~mA}$ does not practically depend on the pulse duration, except for that of $0.7 \mathrm{~ns}$. The latter observation may be attributed to the bandgap thermal shrinking, while the Fermi level stays constant due to recharging of the LED capacitance. Indeed, considering the current magnitude of $\sim 3 \mathrm{~A}$ as the onset of the red shift for $0.7 \mathrm{~ns}$ pulse duration and the "origin" of the linear fit of the maxima of the LED photo response vs pumping current amplitude as shown in Fig. 5, one can estimate the total charge of the "lost" carriers to be of $\sim 2.1 \mathrm{nC}$. This value is comparable by the order of magnitude with the total charge of $\sim 7.1 \mathrm{nC}$ accumulated in either $\mathrm{n}$ - or p-type space-charge region of an unbiased $p-n$ junction at the donor/acceptor concentration of $\sim 3 \times 10^{18} \mathrm{~cm}^{-3}$ and contact potential of nearly $2 \mathrm{~V}$. At other excitation conditions, the point of slope changing in Fig. 1(b) is found to depend on the pulse duration. Assuming the slope change to correspond to the red shift of about $2 \mathrm{~nm}$ [see Fig. 1(b)], one can plot the critical current associated with the slope change as a function of pulse duration $\tau_{p}$ (see Fig. 2). The resulting critical current

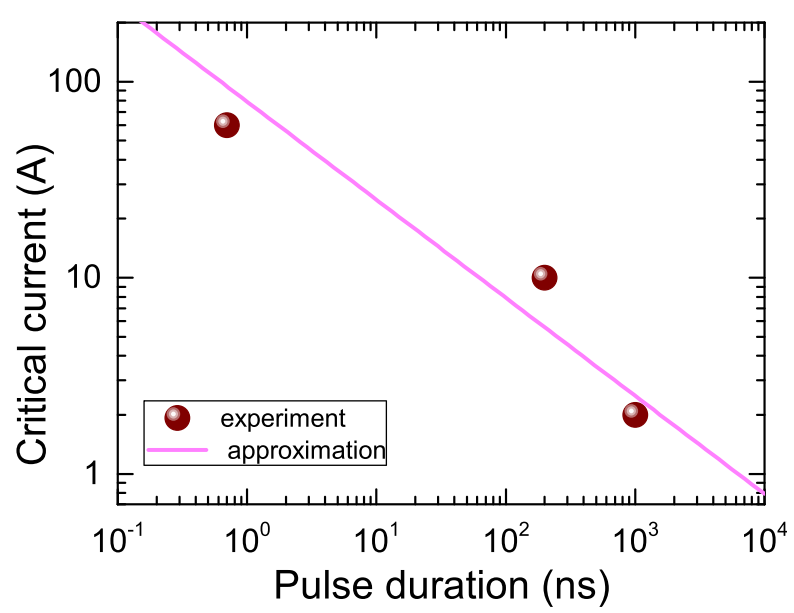

FIG. 2. Critical current $I_{c}$ associated with the slope change as a function of pulse duration $\tau_{p}$. Balls are experimental points, line is approximation assuming $I_{c} \propto \tau_{p}^{-1 / 2}$.

is found to be nearly proportional to $\tau_{p}^{(-1 / 2)}$, which is the evidence for involving thermal effects, i.e., LED self-heating controlled by the pulse duration. Indeed, solution of the heat transfer equation gives for the active region overheating $\Delta \mathrm{T}$ at the end of a rectangular current pulse: $\Delta T \propto I \tau_{p}^{1 / 2}$ where I is the current magnitude. Therefore, in order to reach the overheating $\Delta T_{c}$ corresponding to the red shift of $2 \mathrm{~nm}$, the current magnitude should be varied with the pulse duration as $I_{c} \propto \Delta T_{c} / \tau_{p}^{1 / 2}$. If self-heating becomes important starting from the critical current corresponding to the slope change in Fig. 1(b), a natural question appears: what is the nature of red shift at lower currents?

We believe the red shift to originate from the bandgap shrinking caused by non-equilibrium carrier injection into the LED active region. ${ }^{20}$ In this case, the bandgap narrowing can be estimated as: $\Delta E_{g}=-\gamma(3 N / \pi)^{1 / 3}$ where $\mathrm{N}$ is the electron/ hole concentration in the $\left(A l_{x} G a_{1-x}\right)_{0.52} I_{0.48} P$ QWs, $\gamma=q$ / $2 \pi \epsilon_{0} \epsilon_{s}, \mathrm{q}$ is the elementary charge, and $\epsilon_{0}$ and $\epsilon_{s}$ are the electric and static dielectric constants, respectively. ${ }^{20,21} \mathrm{At}$ $\mathrm{N}=10^{16}-10^{17} \mathrm{~cm}^{-3}$, the above equation provides $\Delta E_{g}=6-$ $13 \mathrm{meV}$, which translates into the red shift of $\sim 2-4 \mathrm{~nm}$ at the wavelength of $620 \mathrm{~nm}$. At higher carrier concentrations, the red shift due to the bandgap shrinking should be compensated by a blue shift caused by the band filling in the QWs. However, contribution of self-heating to the bandgap narrowing does not allow observation of the blue shift.

The two different mechanisms responsible for the red shift in the emission spectra can also be seen in the current dependence of the spectrum width [Fig. 1(c)]. All curves corresponding to different current pulse widths are merged until a critical current is reached, following the increase in the carrier concentration with current due to the band filling effect. Approaching the critical current dependent on the pulse duration (200-600 ns vs $100 \mu$ s and CW excitation) results in a strong additional broadening of the emission spectra, indicating the impact of the LED self-heating. Here, the broadening via band filling effect is accompanied by the broadening related to elevated temperature of non-equilibrium carriers. Behavior of the spectrum width at sub-nanosecond pulse 
excitation cannot be directly compared to those observed at longer current pulses. However, the narrow spectrum widths [Fig. 1(c)] and red shifts smaller than $2 \mathrm{~nm}$ [Fig. 1(b)] measured in this case enable the conclusion that self-heating effects are almost excluded under these pumping conditions despite the very high magnitudes of current supplied.

In order to estimate the LED active region overheating, the temperature dependence of peak emission wavelength is measured under $\mathrm{CW}$ excitation conditions in a wide range of temperatures from $13 \mathrm{~K}$ to $450 \mathrm{~K}$ and at different currents from $20 \mu \mathrm{A}$ to $1 \mathrm{~A}$. A linear coefficient of $7.5 \mathrm{~K} / \mathrm{nm}$ for temperatures $\geq 150 \mathrm{~K}$ is found to be best describing the dependence. This coefficient is close to that of $9.5 \mathrm{~K} / \mathrm{nm}$ reported in Ref. 17. The measurements enabled establishing of the temperature scale [right vertical axis in Fig. 1(b)] for the observed current dependence of the red shift.

\section{B. Efficiency vs current analysis}

Figure 3(b) illustrates the EQE as a function of current $I$, $\mathrm{EQE}(I)$, in CW and sub-microsecond regimes measured in an integrating sphere. Under CW excitation, the gradually increasing $\mathrm{EQE}$ at lower currents attains a maximum at a current of $\sim 60 \mathrm{~mA}$. For currents more than $\sim 200-300 \mathrm{~mA}$, a dramatic decrease in the efficiency is noticed. At $700 \mathrm{~mA} \mathrm{CW}$ pumping, a drop in $\mathrm{EQE}$ of about $15 \%$ of its maximum value is seen. In the sub-microsecond regime, a 15\% drop of $\mathrm{EQE}$ from its maximum value, which corresponds to $\sim 200-250 \mathrm{~mA}$, is observed at $\sim 8-9$ A pump current.

To estimate IQE and LEE under CW operation, the procedure suggested in Ref. 19 based on an AB-model is used. For this purpose, the apparent LEE $\eta_{\text {ext }}$ is calculated by the expression derived straightforwardly from Eq. (1)

$$
\eta_{e x t}=\frac{\left(P_{2}^{1 / 2}-P_{1}^{1 / 2}\right)}{\left(P_{2}^{1 / 2} / \eta_{2}-P_{1}^{1 / 2} / \eta_{1}\right)},
$$

where $\eta_{j}=\eta_{e}\left(P_{j}\right)$ is EQE of the LED measured at the output optical power $\mathrm{P}_{j}$ and $\mathrm{P}_{1}$ and $\mathrm{P}_{2}$ are two different values of the output power. In practice, $\mathrm{P}_{1}$ is chosen from low-current diapason of the $\mathrm{EQE}(\mathrm{I})$ dependence in such a way, as to correspond to $\eta_{e}$ nearly equal to a half of the EQE maximum, whereas $\mathrm{P}_{2}$ passes through all the available experimental points to provide $\eta_{\text {ext }}$ as a function of $\mathrm{P}_{2}$ or corresponding operating current. As a result, $\eta_{\text {ext }}$ has been found to be nearly constant at the currents lower than $0.03 \mathrm{~A}$, where $\mathrm{EQE}$ does not yet tend to saturation [see Fig. 3(a)]. Therefore, this constant value of $\eta_{\text {ext }}$ can be associated with the actual LEE (see Ref. 19 for more details).

General applicability of the recombination model to MQW LED structures is not so obvious because of possible non-uniform carrier distribution among the individual QWs. In order to assess the non-uniformity degree in the case of a red LED MQW structure, we have carried out simulations of its operation with SiLENSe 5.12 package. ${ }^{22}$ A typical LED structure with a $25 \times\left(3.8 \mathrm{~nm} \mathrm{Ga}_{0.52} \mathrm{In}_{0.48} \mathrm{P} /\right.$ $\left.7.0 \mathrm{~nm}\left(\mathrm{Al}_{0.55} \mathrm{Ga}_{0.45}\right)_{0.52} \mathrm{In}_{0.48} \mathrm{P}\right)$ MQW active region has been chosen as a representative case providing a maximum conduction-band offset between the QWs and barriers.
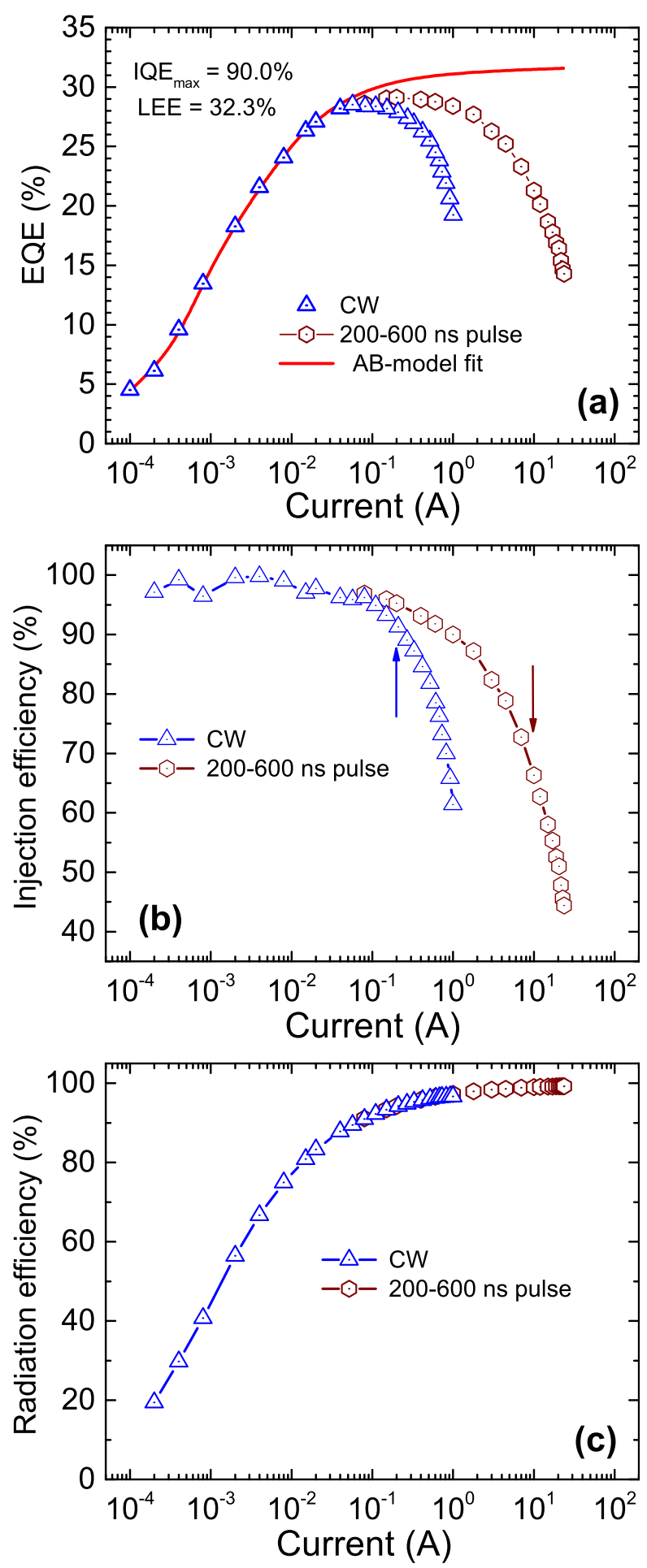

FIG. 3. Current dependence of (a) EQE under sub-microsecond pulses (hexagons) and $\mathrm{CW}$ regime (triangles) where the line shows prediction of the AB-model, neglecting the electron leakage and self-heating effects, (b) injection efficiency, and (c) radiation efficiency under the CW and submicrosecond pulse regime. Arrows in (b) indicate the critical currents at which the dependence of emission wavelength on current changes its slope [see Fig. 1(b)].

Figure 4 displays distributions of electron/hole concentrations and radiative recombination rate (photon emission rate) across the structure, corresponding to an operation current density of $20 \mathrm{~A} / \mathrm{cm}^{2}$. Despite the large number of QWs, the distribution of electron and hole concentrations are found to 

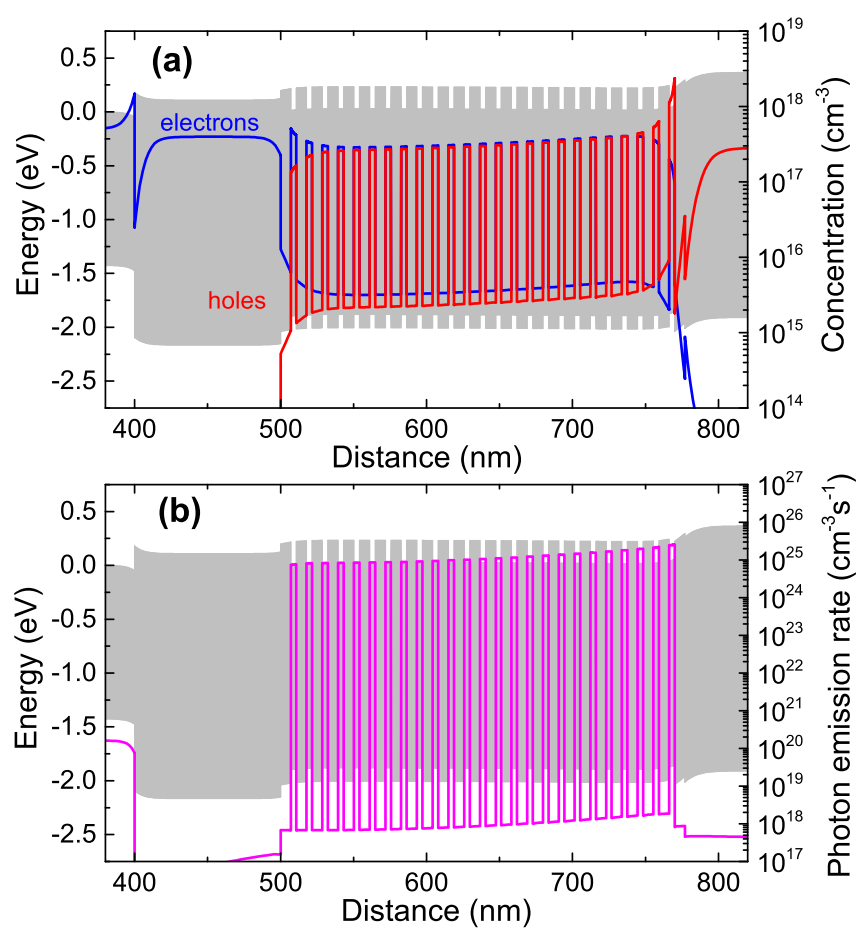

FIG. 4. Simulated room-temperature band diagram and distributions of electron and hole concentrations (a) and photon emission rate (b) in a typical red LED structure with the $25 \times\left(3.8 \mathrm{~nm} \mathrm{Ga}_{0.52} \mathrm{In}_{0.48} \mathrm{P} / 7.0 \mathrm{~nm}\left(\mathrm{Al}_{0.55} \mathrm{Ga}_{0.45}\right)_{0.52} \mathrm{In}_{0.48} \mathrm{P}\right)$ MQW active region operating at the current density of $20 \mathrm{~A} / \mathrm{cm}^{2}$. The grey shadow indicates the bandgap.

be rather uniform, except for QWs placed at the edges of the active region [Fig. 4(a)]. However, even here, the concentration varies in such a way, as not to disturb the uniform distribution of the radiative recombination rate across the structure [Fig. 4(b)]. At higher current densities, all the distributions become even more uniform. The simulations show also that contribution of Auger recombination in the LED active region to the total recombination rate does not exceed $3 \%$ up to the current densities of $10 \mathrm{kA} / \mathrm{cm}^{2}$. These results justify the application of the AB-recombination model for estimation of IQE and LEE from the characterization data, especially if the carrier concentration considered in the model is defined as the geometrical mean of the actual concentrations of electrons and holes.

Light extraction efficiency $\left(\eta_{\text {ext }}\right)$ of $32.3 \%$ and optical power at $50 \%$ IQE $\left(P_{0.5}\right)$ of $0.435 \mathrm{~mW}$ (see Ref. 20 for more detailed explanation of the parameters) are estimated at low operation currents up to $\sim 10-30 \mathrm{~mA}$. These currents correlate well with the onset of the red shift in the LED emission spectra plotted in Fig. 1(b). Measurements of EQE of the LED at various temperatures under $\mathrm{CW}$ operation and application of the fitting procedure ${ }^{19}$ have shown that $P_{0.5}$ is practically constant in the temperature range between 300 and $350 \mathrm{~K}$. This enables estimating the radiation efficiency of the LED active region using the $\mathrm{AB}$ recombination model ${ }^{19}$ as follows: $\eta_{R}=\left[\sqrt{P_{0.5} / P_{\text {out }}}+1\right]^{-1}$. Where $\mathrm{P}_{\text {out }}$ is the LED output power. Then, IQE $=\mathrm{EQE} / \eta_{\text {ext }}$ and $\mathrm{IE}=\mathrm{IQE} / \eta_{R}$ characterizing the strength of electron leakage is obtained and shown in Fig. 3(b).

$\mathrm{EQE}(\mathrm{I})$ as predicted by the reduced AB-model, assuming (a) isothermal conditions of operation and (b) no electron leakage, is shown in Fig. 3(a) by the dashed line. A maximum IQE value of $\sim 90 \%$ is estimated for the $\mathrm{CW}$ regime from the experimental EQE and estimated LEE. A relatively low maximum EQE value of $\sim 29 \%$ is attributed to an insufficiently high LEE of $32.3 \%$, as expected from the device in the absence of the silicone lens cover. The AB-model is in good agreement with the experimental data at low currents in the CW regime, neglecting Auger recombination.

Radiation efficiency [Fig. 3(c)] is found to grow with current irrespective of the kind of the active region pumping. It saturates at $\sim 100 \%$ when the current approaches the value of $\sim 5-7 \mathrm{~A}$. As IQE of the LED is the product of IE and $\eta_{R}$, the maximum IQE value is limited by the IE decline at high currents.

Up to the current of $\sim 0.1 \mathrm{~A}$, IEs of the LED under CW and sub-microsecond excitation are nearly the same [see Fig. 3 (b)]. Starting from the critical current of $\sim 0.2 \mathrm{~A}$, IEs determined for CW operation decrease dramatically with current, which can be attributed to the device self-heating. On the other hand, sub-microsecond-pulse pumping substantially increases the IE at high currents even though there is a remarkable decline in the IE at currents greater than $\sim 1 \mathrm{~A}$ [Fig. 3(b)]. The decline becomes much more pronounced when the critical current of the nanosecond excitation ( $\sim 10 \mathrm{~A})$ is approached [Fig. 3(b)]. Such a behavior allows us to conclude that both high-carrier concentration and thermal effects are responsible for the IE decrease at high-current operation conditions.

To mitigate the self-heating effects, the LED is then operated in the pulse regime using $\sim 0.7 \mathrm{~ns}$ pulses (FWHM) at $10 \mathrm{kHz}$ repetition rate for very high currents up to $45 \mathrm{~A}$. The experimental maxima of the LED photo response are well fitted linearly when plotted against the pumping current magnitude (shown as a blue dashed line in Fig. 5). It is interesting that the approximation line crosses the current axis at

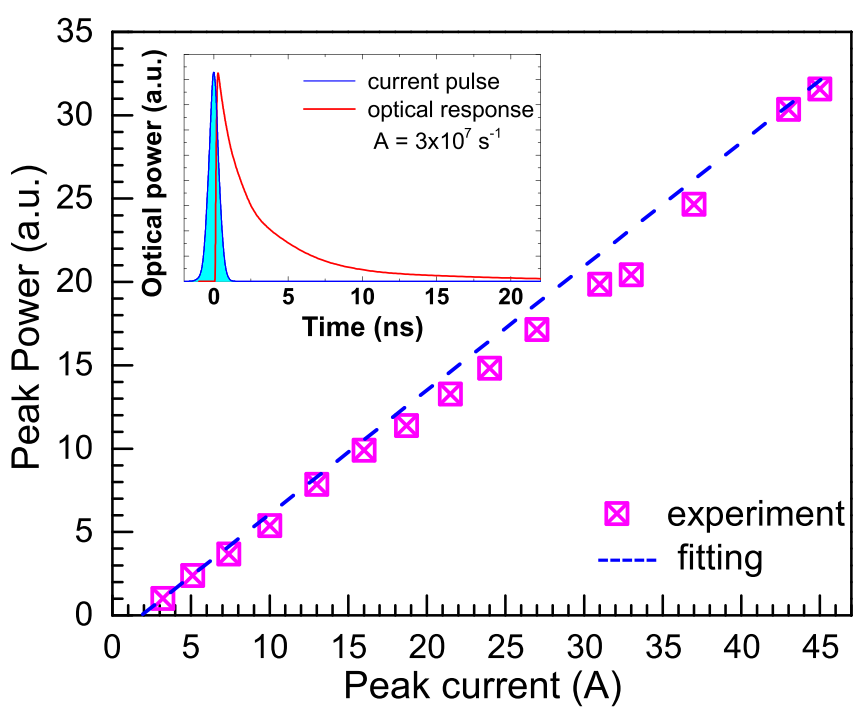

FIG. 5. Peak power behavior under wide range of $0.7 \mathrm{~ns}$ peak currents. The magenta squares represent the experimental data and linear fitting is depicted by the blue dashed line. The inset present results of time-resolved EL simulations, assuming the Shockley-Read-Hall recombination constants $\mathrm{A}=3 \times 10^{7} \mathrm{~s}^{-1}$ : the green shadow shows a Gaussian current pulse and the red solid line indicates the photo response. 
$\sim 3$ A. The observation of the photo response onset justifies the idea of partial losses of carriers supplied by a short current pulse for recharging the space-charge regions in the LED structure.

The linear dependence of EL intensity on the current magnitude in Fig. 5 would suggest at first glance that EQE is unaffected by current. However, simulations of the timedependent EL of the LED have shown that the duration of the optical response is remarkably longer than that of the subnanosecond current pulse (see the inset in Fig. 5). This requires reconsideration of the usual method of IQE and, hence, EQE determination, as well as finding a reasonable way for the efficiency comparison at sub-nanosecond and CW excitation. This part of our study will be reported elsewhere.

\section{CONCLUSION}

In summary, high power commercial red LEDs have been investigated under $\mathrm{CW}$ and pulsed current pumping. Monitoring of current $I_{o}$ corresponding to the onset of the red shift in the emission spectra (independent of pulse duration) and critical current $I_{c}$ corresponding to the change in the slope of the spectral red shift (dependent on the pulse width) and finding correlation between $I_{o}\left(I_{c}\right)$ and saturation (drop) of the LED efficiency with current allows distinguishing between various operation conditions. At $\mathrm{I}<I_{o}$, the increase in carrier concentration in the active region and self-heating do not practically influence the LED operation, so that the LED efficiency strictly follows the theoretical predictions made by the reduced ABC-model. At $I_{o}<I<I_{c}$, electron leakage into p-side of the LED structure is induced due to a high concentration of carriers injected into the active region, resulting in the efficiency saturation and a weak red shift of the emission spectra due to bandgap shrinking. At $I$ $>I_{c}$, device self-heating starts to dominate, enhancing the electron leakage and resulting in a dramatic efficiency decrease and a much stronger red shift of the emission spectra, accompanied by their additional broadening. In this case, the estimated overheating of the LED active region may approach $\sim 80 \mathrm{~K}$ under $\mathrm{CW}$ operation.

Using short current pulses for LED pumping enables substantially increasing the threshold current $\left(I_{c}\right)$ of the device self-heating without noticeable losses of the emission efficiency. In particular, $I_{c}$ can be increased by about two orders of magnitude compared to $\mathrm{CW}$ regime by utilizing sub-microsecond current pulses. This enables obtaining a high LED efficiency at much higher current magnitudes, as compared to CW LED operation. The use of sub-nanosecond excitation almost completely avoids the LED self-heating even at the currents as high as $\sim 45 \mathrm{~A}$. However, the latter case requires a more elaborated examination because of essentially different durations of the current pulse and photo response. Development of a corresponding approach is one of the tasks for future studies. From the practical point of view, the use of sub-microsecond current pulses for LED excitation enables increasing the current by the order of magnitude, as compared to $\mathrm{CW}$ excitation, without noticeable losses in the emission efficiency.
The estimated maximum IQE value ( $~ 90 \%$ at $60 \mathrm{~mA}$ under CW pumping) and LEE $(\sim 32 \%)$ of the studied red LED suggest that light extraction from the LED die still remains the bottleneck for the overall emission efficiency rather than the electron leakage enhanced by both high carrier concentration in the active region and device selfheating. Indeed, even at $700 \mathrm{~mA}$ the IQE decreases just by 13.5 absolute percent. On the other hand, LEE of 90\% has been demonstrated to be achievable for nitride LEDs. ${ }^{23}$ So, the room for LEE improvement from $\sim 32 \%$ to $\sim 90 \%$ is remarkably larger than the IQE losses caused by the electron leakage. According to estimations made in Ref. 23, recent progress in the LED fabrication technology ${ }^{24}$ has improved LEE of red LEDs, up to $65 \%$. Nevertheless, this level is still lower than that achieved in III-Nitride LEDs.

\section{ACKNOWLEDGMENTS}

This work was supported by the European Union FP7 NEWLED project (Grant No. 318388). This work was also partially supported by the Grant of Russian Scientific Foundation 18-15-00172.

\section{APPENDIX: AB-RECOMBINATION MODEL FOR LIGHT EXTRACTION EFFICIENCY EVALUATION}

The AB-recombination model considers two competitive recombination channels: Shockley-Read-Hall non-radiative recombination with the corresponding recombination coefficient $\mathrm{A}$ and bimolecular radiative recombination with the coefficient B. Within the model, the current I flowing through an LED and the corresponding output optical power $\mathrm{P}_{\text {out }}$ are determined by the expressions

$$
I=q V_{r}\left(A n+B n^{2}\right) / \eta_{\text {inj }}, \quad P_{\text {out }}=E_{p} V_{r} \eta_{\text {ext }} B n^{2},
$$

where $\mathrm{n}$ is the electron or hole concentration in the LED active region, assumed to be nearly equal to each other, $\mathrm{q}$ is the elementary charge, $\mathrm{V}_{r}$ is the recombination volume, i.e., a part of or the whole volume of the active region where intensive recombination occurs, $E_{p}$ is the mean energy of emitted photons, and $\eta_{i n j}$ and $\eta_{\text {ext }}$ are the injection efficiency and LEE, respectively; LEE is conventionally assumed to be independent of current. EQE of LED $\eta_{e}$ is defined by the following relationships:

$$
\begin{aligned}
\eta_{e} & =\left(\frac{q}{E_{p}}\right) \frac{P_{\text {out }}}{I}=\eta_{\text {ext }} \eta_{\text {inj }} \frac{B n}{A+B n}=\eta_{\text {ext }} \eta_{\text {inj }} \frac{n}{n_{0.5}+n} \\
& =\eta_{\text {ext }} \eta_{\text {inj }} \frac{P_{\text {out }}^{1 / 2}}{P_{0.5}^{1 / 2}+P_{\text {out }}^{1 / 2}} .
\end{aligned}
$$

Here, $n_{0.5}=A / B$ is the specific carrier concentration dependent on particular recombination coefficients and $P_{0.5}$ is the output power corresponding to $n=n_{0.5}$. The fraction in the right side of Eq. (A2) represents the radiative efficiency $\eta_{R}$ relevant to recombination processes only. One can see that $\eta_{R} \rightarrow 1$ when $P_{\text {out }} \rightarrow \infty$.

If the carrier leakage from the LED active region is absent, which is the case of low currents, $\eta_{i n j}=1$ and 
Eq. (A2) can be reduced to Eq. (1) given in Sec. I. Considering two EQE values, $\eta_{1}$ and $\eta_{2}$, that correspond to different output optical powers $P_{1}$ and $P_{2}$, one can exclude the parameter $P_{0.5}$ and obtain Eq. (2) in Sec. IV B, which provides at low currents the actual value of LEE. In a similar manner, the power $P_{0.5}$ can be also evaluated (see Ref. 19 for more details).

${ }^{1}$ T. Gessmann and E. Schubert, J. Appl. Phys. 95, 2203 (2004).

${ }^{2}$ J. Y. Tsao, M. H. Crawford, M. E. Coltrin, A. J. Fischer, D. D. Koleske, G. S. Subramania, G. T. Wang, J. J. Wierer, and R. F. Karlicek, Adv. Opt. Mater. 2, 809 (2014).

${ }^{3}$ M. Crawford, IEEE J. Sel. Top. Quantum Electron. 15, 1028 (2009).

${ }^{4}$ D. A. Vanderwater, I. H. Tan, G. E. Höfler, D. C. Defevere, and F. A. Kish, Proc. IEEE 85, 1752 (1997).

${ }^{5}$ D. Patel, J. M. Pikal, C. S. Menoni, K. J. Thomas, F. A. Kish, and M. R. Hueschen, Appl. Phys. Lett. 75, 3201 (1999).

${ }^{6}$ P. Altieri, A. Jaeger, R. Windisch, N. Linder, P. Stauss, R. Oberschmid, and K. Streubel, J. Appl. Phys. 98, 086101 (2005).

${ }^{7}$ J. Cho, E. F. Schubert, and J. K. Kim, Laser Photonics Rev. 7, 408 (2013).

${ }^{8}$ J.-W. Shi, F.-M. Kuo, C.-W. Lin, W. Chen, L.-J. Yan, and J.-K. Sheu, IEEE Photonics Technol. Lett. 23, 1585 (2011).

${ }^{9}$ C.-F. Huang, Y.-F. Su, C.-B. Lin, and K.-N. Chiang, Solid State Electron. 93, 15 (2014)

${ }^{10}$ J.-I. Shim, D.-P. Han, H. Kim, D.-S. Shin, G.-B. Lin, D. S. Meyaard, Q. Shan, J. Cho, E. Fred Schubert, H. Shim, and C. Sone, Appl. Phys. Lett. 100, 111106 (2012)
${ }^{11}$ R. Windisch, P. Altieri, R. Butendeich, S. Illek, P. Stauss, W. Stein, W. Wegleiter, R. Wirth, H. Zull, and K. Streubel, Proc. SPIE 5366, 43 (2004).

${ }^{12}$ P. Royo, R. P. Stanley, M. Ilegems, K. Streubel, and K. H. Gulden, J. Appl. Phys. 91, 2563 (2002).

${ }^{13}$ Z. Gong, S. Jin, Y. Chen, J. McKendry, D. Massoubre, I. M. Watson, E. Gu, and M. D. Dawson, J. Appl. Phys. 107, 013103 (2010).

${ }^{14}$ P. Tian, J. J. D. McKendry, Z. Gong, S. Zhang, S. Watson, D. Zhu, I. M. Watson, E. Gu, A. E. Kelly, C. J. Humphreys, and M. D. Dawson, J. Appl. Phys. 115, 033112 (2014).

${ }^{15}$ N. Lobo Ploch, H. Rodriguez, C. Stolmacker, M. Hoppe, M. Lapeyrade, J. Stellmach, F. Mehnke, T. Wernicke, A. Knauer, V. Kueller, M. Weyers, S. Einfeldt, and M. Kneissl, IEEE Trans. Electron Dev. 60, 782 (2013).

${ }^{16}$ H. X. Zhang, D. Massoubre, J. McKendry, Z. Gong, B. Guilhabert, C. Griffin, E. Gu, P. E. Jessop, J. M. Girkin, and M. D. Dawson, Opt. Express 16, 9918 (2008).

${ }^{17}$ P. Manninen and P. Orreveteläinen, Appl. Phys. Lett. 91, 181121 (2007).

${ }^{18}$ C. Van Opdorp and G. W. Hooft, J. Appl. Phys. 52, 3827 (1981).

${ }^{19}$ A. Yadav, I. E. Titkov, G. S. Sokolovskii, S. Y. Karpov, V. V. Dudelev, K. K. Soboleva, M. Strassburg, I. Pietzonka, H.-J. Lugauer, and E. U. Rafailov, Proc. SPIE 9768, 97681K (2016).

${ }^{20}$ P. A. Wolff, Phys. Rev. 126, 405 (1962).

${ }^{21}$ B. R. Bennett, R. A. Soref, and J. A. Del Alamo, IEEE J. Quantum Electron. 26, 113 (1990).

${ }^{22}$ STRGroup, see http://www.str-soft.com/products/SiLENSe/index.htm for SiLENSe-software tool for light emitting diode (LED) bandgap engineering (last accessed 02-Feb-2018).

${ }^{23}$ S. Y. Karpov, Proc. SPIE 9768, $97680 \mathrm{C}$ (2016).

${ }^{24}$ M. Broell, P. Sundgren, A. Rudolph, W. Schmid, A. Vogl, and M. Behringer, Proc. SPIE 9003, 90030L (2014). 